\title{
Altering barley agronomic traits via targeted mutagenesis
}

\author{
S.V. Gerasimova ${ }^{1, *}$, A.M. Korotkova ${ }^{1}$, C. Hertig ${ }^{2}$, I. Otto ${ }^{2}$, S. Hiekel², J. Kumlehn², \\ A.V. Kochetov ${ }^{1,3}$, E.K. Khlestkina ${ }^{1,3,4}$ \\ ${ }^{1}$ Institute of Cytology and Genetics SB RAS, Novosibirsk, Russia \\ ${ }^{2}$ Leibniz Institute of Plant Genetics and Crop Plant Research (IPK), Gatersleben, Germany \\ ${ }^{3}$ Novosibirsk State University, Novosibirsk, Russia \\ ${ }^{4}$ N.I. Vavilov All-Russian Research Institute of Plant Genetic Resources (VIR), Saint-Petersburg, Russia \\ *e-mail: gerson@bionet.nsc.ru
}

Key words: barley, Hordeum vulgare, genome editing, crop improvement, Cas9, gRNA, spike architecture

Motivation and aim: Target-specifically customizable endonucleases are a new molecular tool, which opens a huge variety of novel opportunities for life sciences, biotechnology and breeding. Crop improvement is one of the most promising applications of this technology. The aim of study is to modify agronomical traits of barley (Hordeum vulgare L.) exploiting RNA-guided endonuclease. Targeted knockout of Nud and Vrs 1 genes should lead to phenotype switches from hulled to naked barley and two-rowed to six-rowed spike, respectively. To extend the utilization of the technology from the model to local elite cultivars, we decided to evaluate the regeneration ability of immature embryos from the ten most prospective spring barley cultivars of the Siberian collection to include the best performing ones in our approach.

Materials and Methods: In vitro multiple shoot formation was tested across several barley cultivars and lines ("Biom", "Talan", "Vorsinskiy 2", "Aley", "Acha", "Signal", "L-421", "Kolchan", "V-1", "Krasnoyarskiy 91", "Golden Promise"). The structure of Nud and Vrs1 genes was confirmed by sequencing. Adequate guide RNAs for targeting Nud and Vrsl barley genes were selected with available online-tools. Transformation vectors with different gRNAs and Cas9 genes were assembled for transient and stable expression in barley. The activity of gRNAs was tested in vivo using the methods of mutational restoration of reporter gene functionality [1] and protoplast transfection with subsequent T7E1 assay [2]. Selected genetic constructs were introduced into the barley genome by Agrobacterium-mediated gene transfer to immature embryos.

Results: Regeneration analysis of Siberian barley cultivars revealed few cultivars with high in vitro regeneration capacity. Cultivar "Aley" was selected for further experiments aiming at site-directed mutagenesis. A set of different gRNAs for knockout of $\mathrm{Nud}$ and Vrs 1 genes was established. In silico and in vivo evaluation of gRNA activity revealed highly active gRNAs for both genes. Genetic constructs containing gRNA and Cas9 expression cassettes were introduced into the genome of barley and primary transgenic plants were obtained.

Conclusion: RNA-guided endonuclease targeting Nud and Vrs 1 genes was shown to be highly active in barley cells of cv. "Aley" and "Golden Promise", genetic transformation of the Siberian cv. "Aley" is being established.

Acknowledgements: The study is supported by the RSF (No. 16-14-00086).

\section{References}

1. Budhagatapalli N. et al. (2016) A simple test for the cleavage activity of customized endonucleases in plants. Plant Methods. BioMed Central. 12(1):1-10.

2. Shan Q. et al. (2014) Genome editing in rice and wheat using the CRISPR/Cas system. Nat. Protoc. 9:2395-2410. 